\title{
Mesh Scalability Concept for Explicit Simulation of Rock Failure
}

\author{
A.V. Dyskin School of Civil and Resource Engineering, The University of Western Australia, Australia
}

A. Caballero Swiss Federal Institute of Technology of Lausanne, Switzerland

\begin{abstract}
Rock failure is a phenomenon which involves the initiation and propagation of (multiple) fractures and the development of surfaces of strain localisation. Direct numerical simulation of these phenomena by introducing the relevant constitutive behaviour in finite elements (or similar computational elements, e.g. particles in the discrete element method) lead to mesh dependence: the computations with successively refined meshes do not stabilise. The mesh dependence is caused by the fact that theoretically, fractures and strain localisation surfaces have zero thickness, while in numerical calculations this thickness is finite controlled by the size of the finite element or particle, or by a characteristic size of the non-local functions in mesh-free methods. Two methods are currently used to overcome this problem. The first is the introduction of a generalised continuum which contributes its own characteristic lengths. The computations stabilise as soon as the size of the computational element becomes considerably smaller than the smallest characteristic length of the continuum. The use of generalised continua as numerical stabilisers leads however to hard questions as to what type of the continuum is to be chosen and how to determine its numerous parameters. In the second method a size of the mesh element is chosen which corresponds to the size of microstructural elements (if this size in known). In this approach the flexibility of choosing computationally efficient element size is lost if the microstructural elements are very small compared with the characteristic size of the geometry to be modelled (e.g. the excavation size) the modelling may become computationally prohibitive, exactly as in the case of particle methods. To deal with mesh dependence we propose the concept of mesh scalability. We use the assumption that, if the element size is sufficiently small, all mesh-dependent quantities should depend upon the size as power functions. The mesh with this property is called scalable and is regarded as sufficiently fine for the simulations. The obtained exponent and the pre-factor can be used to extrapolate the mesh dependent quantities to the actual microstructural sizes. Furthermore, the method permits the identification of stress singularities from finite element computations with different element sizes in similar meshes. The pre-factors are then called the mesh stress intensity factors and can be used to analyse failure instead of the ones referred to in the Linear Fracture Mechanics. We consider examples of application of this philosophy to modelling the stability of rock slopes.
\end{abstract}

\section{Introduction}

Rock failure is a phenomenon which involves the initiation and propagation of (multiple) fractures and the development of surfaces of strain localisation. Direct numerical simulation of these phenomena by introducing the relevant constitutive behaviour in finite elements (or similar computational elements, e.g. particles in the LSM) lead to mesh dependence (Bazant et al., 1984; de Borst et al., 1993): the computations with successively refined meshes do not stabilise. The mesh dependence is caused by the fact that theoretically, fractures and strain localisation surfaces have zero thickness, while in numerical calculations this thickness is finite and being controlled by either the size of the discretisation element (the element of the mesh in finite element or finite difference methods or particle), or by a characteristic size of the non-local functions in mesh-free methods. Two approaches are currently employed to overcome this problem. The first is the introduction of a (generalised) continuum whose constitutive relationship possesses characteristic lengths (Sluys et al., 1993). Then the computations stabilise as soon as the size of the computational element becomes considerably smaller than the smallest characteristic length of the continuum. While the use of generalised continua is justified in the cases where the additional internal degrees of freedom (e.g. independent rotations (Pasternak et al., 2001)) are important, their usage as numerical stabilisers leads to hard questions as to what type of the continuum has to be chosen and how to determine its numerous parameters. The second approach puts forward a mesh whose element size corresponds to the size of 
microstructural elements (assuming that this size in known). In this approach the flexibility of choosing a computationally efficient element (particle) size is lost. In particular, if the microstructural elements are very small compared to the characteristic size of the geometry to be modelled (e.g. the excavation size) the modelling may become computationally prohibitive, as in the case of particle methods.

In order to deal with mesh dependence, rather than dropping the finite element-type methods in favour of direct fracture mechanics simulations involving crack propagation or the displacement discontinuity approach (Klein et al., 2001) which would bring new questions about the location, growth and the type of behaviour of the introduced cracks or discontinuities, we propose the concept of mesh scalability. Generally we suggest quantifying the dependence of the computed quantities upon the (minimal) element size. As a particular case we use the assumption that, if the element size is sufficiently small, all mesh-dependent quantities should depend upon this size as power functions. The mesh with this property is called scalable and is regarded as sufficiently fine for the simulations. This philosophy is explained in Section 2 . The obtained exponent and the pre-factor can then be used to extrapolate the mesh dependent quantities to the actual microstructural sizes. The method also permits the identification of stress singularities from finite element computations with different element sizes in similar meshes. The pre-factors can then be used to analyse failure instead of the ones used in Linear Fracture Mechanics. This is discussed in Section 3. Section 4 considers an example of application of the proposed method to the stability of open pit.

\section{The concept of mesh scalability}

As indicated in the Introduction, mesh dependence in geotechnical modelling is dealt with either by explicit introduction of a stabilising length scale or by associating this length with the size of the chosen discretisation (Tang, 1997). In the former case the major difficulty is the identification of such a length scale. If the problem is solved when, for instance, other thermodynamics processes are included in the model description naturally producing the length scale then the problem of mesh dependence is solved (RegenauerLieb and Yuen, 2000). In the latter case the mesh dependence is not actually solved, just a certain mesh is declared to be appropriate. One can of course argue that if the size of the finite element is made equal to the size of the microstructural element of the geomaterial in question, then the model will adequately describe the behaviour of the geotechnical structure. However, there are three issues with such an approach. Firstly this choice of the element size does not guaranty the numerical accuracy. Indeed, in conventional finite element modelling the mesh is eventually chosen such that further refinement does not lead to any further variations in the solution. This means that the element size is already small as compared to both mechanically and numerically induced internal scales (the latter being essentially numerical artefacts are nevertheless present in numerical models). In the mesh dependent situations there is no way to find a mesh size to ensure the numerical accuracy. Secondly, the results of numerical modelling also depend upon the type of elements. Again, in the absence of a criterion of numerical stability the 'right' type of element cannot be identified as the real microstructural elements cannot lend any guidance. The third issue is logistic: what if the microstructural elements are too small leading to computationally prohibitive meshes? This problem is especially apparent in the case when a discrete element method of a sort in which the discrete element represents a grain of the rock or soil. The sheer numbers of grains involved in real size geotechnical structure is simply too large to be computationally tractable. (An apparent shortcut - the introduction of super particles or grains will immediately lead to mesh, or rather grain size dependence.)

In this paper we propose a different way to deal with mesh dependence. We firstly recognise that the mesh dependence is a natural outcome of the introduction of an additional characteristic length to the problem the finite element or particle size which was not present in the original mathematical formulation. Once introduced, this additional size interacts with the other characteristic lengths present in the formulation. We consider here a special case of such interaction which we will call the separation of scales. This is the case in which the element size can be chosen small enough such that its stress-strain state became independent upon other characteristic lengths of the problem in the following sense. We assume that one can choose such a small region around a point (or the element) of interest such that the parameters, including characteristic lengths of the stress-strain distribution, are solely determined by the size of the element. Then the dependence of these parameters on the element size $h$ is expressed by the power law:

$$
\sigma(h)=S h^{\alpha}
$$


Here $\sigma$ is a parameter of stress-strain distribution, $\alpha$ is the exponent and $S$ is a pre-factor. The reason that dependence upon $h$ can only assume the form of a power law is because the only argument of this function, the element size, is a dimensional quantity. The dependence as such should be independent of free units chosen to measure $h$; this brings the possible choice of the function expressing this dependence to the power law (see Barenblatt and Botvina, 1980; Gelikman and Pisarenko, 1989; Zosimov and Lyamishev, 1995 for a rigorous proof).

Figure 1 illustrates possible types of behaviour of $\sigma$ in the process of mesh refinement as related to dependence Equation (1).
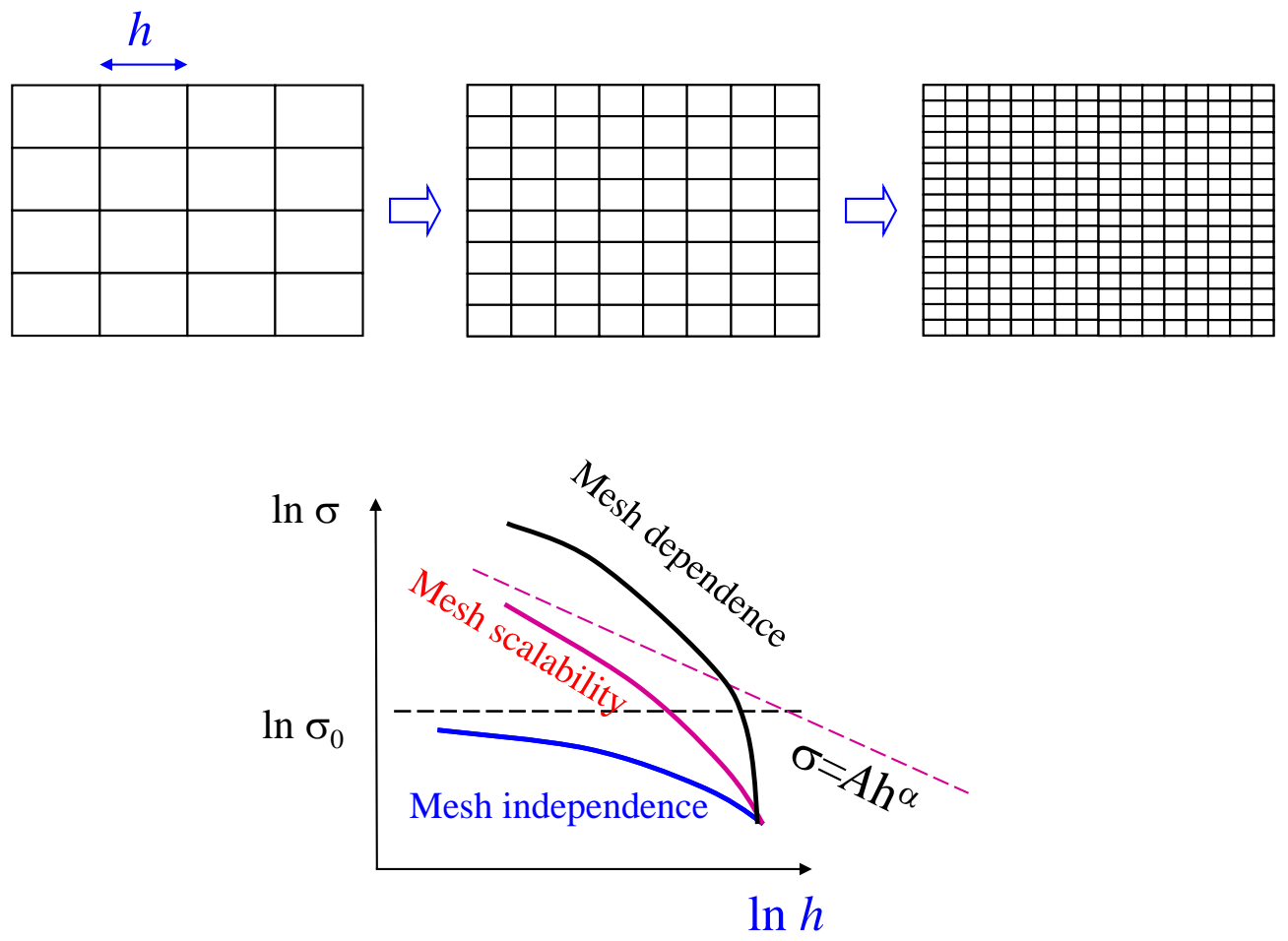

Figure 1 Log-log representation of possible types of dependence of the parameter of stress-strain state $\sigma$ upon the element size in the process of mesh refinement: mesh independence, $\alpha=0$, which is the classical situation; mesh scalability when the power law behaviour is attained and the mesh dependence types not covered by the presented approach

The first case is the case of mesh independence when the solution stabilises. In terms of Equation (1) this case is characterised by zero exponent, $\alpha=0$. The second case is an asymptotic approach to Equation (1). If this is the case, then when the dependence upon $h$ becomes close enough to Equation (1), as far as numerical method is concerned the mesh is fine enough since the mesh dependence is now quantified. We call the mesh at the corresponding state of refinement scalable. As soon as this mesh is found, the results of the computation can be scaled using Equation (1) to any appropriate element size. This essentially disentangles the numerical method from the microstructural size: the mesh size is chosen by computational considerations, approaching dependence Equation (1), no matter how small the microstructural element can be. Furthermore, the following section will propose a method of utilising the information hold by the prefactor. The third case, the case when Equation (1) cannot be achieved represents other types of mesh dependence. This case surely exists, an example being the problem of a rigid punch without slipping on an elastic half-plane (represents a rigid foundation on a soft ground) when the stress singularity cannot be expressed in a power law form, however dealing with them is beyond the scope of the present contribution.

We now concentrate on mesh scalability. We consider a particular case when the mesh dependence is associated with the presence of stress singularities in the original elastic problem. Then, obviously, the exponent $\alpha$ in the power law Equation (1) coincides with the singularity exponent. Then the scalable mesh 
allows one to recover the singularity exponent as well. The question is then what information can be extracted from the value of pre-factors determined in the process of attaining mesh scalability. The next section proposes an interpretation of pre-factors as mesh-related stress intensity factors of a sort. This interpretation can be used to compare different geotechnical scenarios in terms of stability against failure.

\section{Interpretation of pre-factors in the case of stress singularities}

Consider the case when the elastic model provides sufficiently accurate description of the geotechnical structure under consideration and consider its finite element approximation. If the elastic model possesses stress singularities, i.e. infinite stresses (e.g. at the crack tip, if the fractures are involved or at a corner) then the finite element model will show mesh dependence as the stresses at the elements which correspond to the points of stress singularity would tend to infinity as their size tends to zero. Suppose that the stress singularities can be described by power laws as in the case of cracks or corners. Then the finite element model will permit scalable meshes. Let such a mesh be attained.

Suppose that failure initiation is determined by a local fracture criterion at a point $\mathbf{x}$ of the material. We express the failure initiation criterion in the following form:

$$
\sigma_{\max }=\sigma_{c}
$$

where $\sigma_{c}$ is the local strength of a material element $V_{h}$ which correspond to the finite element of size $h$ and

$$
\sigma_{\max }=\max _{\mathbf{x} \in B} F\left(\sigma_{1}(\mathbf{x}), \sigma_{2}(\mathbf{x}), \sigma_{3}(\mathbf{x})\right)
$$

Here $\sigma_{1}(\mathbf{x}), \sigma_{2}(\mathbf{x}), \sigma_{3}(\mathbf{x})$ are the principal stresses at point $\mathbf{x}$ and $F$ is a function which represents the local fracture criterion. This function is homogeneous of the first degree, which follows from the dimensional analysis.

Suppose the loading of the geotechnical structure is controlled by a single loading parameter $P$. (For instance, it could be the gravitational load, the stress of uniaxial compression or tension, the magnitude of hydrostatic pressure or the indentation force.) Then, due to elasticity the maximum force should linearly depend upon $P$ such that:

$$
\sigma_{\max }=S \cdot P
$$

Substituting (4) into (2) one can determine the magnitude of failure load $P_{\text {fail }}$ :

$$
S \cdot P_{\text {fail }}=\sigma_{c}
$$

By definition of mesh scalability,

$$
\sigma_{\text {max }}(h)=A \cdot h^{-\alpha}, \quad \alpha \geq 0
$$

or in terms of Equation (4), since $P$ is the loading parameter which can be held constant

$$
S(h)=B \cdot h^{-\alpha}, \quad \alpha \geq 0
$$

substituting (7) into (5) one obtains for an expression for the failure load

$$
P_{f a i l} B \cdot h^{-\alpha}=\sigma_{c}(h)
$$

The critical stress $\sigma_{c}(h)$ is now mesh-dependent. It is associated with the strength of a finite element and will be called the associated strength. From here the failure load can be expressed as

$$
P_{\text {fail }} B=\sigma_{c}(h) h^{\alpha}
$$

The failure load $P_{\text {fail }}$ is believed to exist objectively and hence is independent upon the mesh size. The prefactor $B$ is independent of $h$ as well, but might depend upon the type of elements used. Therefore, the combination: 


$$
N_{c}=\sigma_{c}(h) h^{\alpha}
$$

is scale invariant (in the sense of scaling associated with the change of mesh size), i.e. mesh independent. It may however depend upon the type of elements chosen.

The mesh scalability essentially means that the geometric dimensions of the body do not influence the form of mesh dependence of the maximum stress (in the sense that it approaches the power law form). Hence, $N_{c}$ is independent of the body geometry. It can therefore be regarded as a property of the 'geomaterial' (more exactly, its finite element representation), depending on the type of loading and the environment (temperature, moisture, etc) and, because it is associated with the finite element representation, upon the mesh configuration such as mesh density distribution and the type of elements. If the type of local loading is kept the same (e.g. local uniaxial tension) then $N_{c}$ is a constant and once being experimentally (or numerically) determined for one geometry it can be used for other configurations as long as the exponent $\alpha$ is the same.

In a way, the stress invariant $N_{c}$ is analogous to the generalised stress intensity factors (Severyn and Molski, 1996), however there is an important difference: as $N_{c}$ is related to a particular type of mesh it is not equal to the corresponding generalised stress intensity factor but rather proportional to it. Finding the proportionality factor can be a computationally intensive task. Instead we propose to use the stress invariant $N_{c}$ in its own right. The motivation behind this proposal is simple. The genuine stress intensity factors only represent the singularity feature of the elastic solution: the real materials do not support infinite stresses. The stress intensity factors are in essence used to compare, in a nice and simple form, the results of the elastic computations with stress intensity factors calculated for the samples at the fracture initiation in the fracture mechanics experiments. This comparison forms the experimental basis for the fracture propagation criteria, which are essentially the criteria of local failure. For the classical fracture mechanics problems, this comparison is possible because the singularity exponent is -0.5 , which is the same in all elastic problems involving cracks. For other geometries which involve stress singularities with other exponents, similar local failure criteria are only possible within the situations characterised by the same exponent. In particular in the case of corners this would correspond to the situations of the same corner angle and the same type of boundary conditions. In the spirit of this remark one can achieve the same with the stress invariant $N_{c}$. We will call it the Mesh Stress Intensity Factor to emphasise its dependence upon the type of the mesh. Indeed for the cases characterised by the same exponent one can compare the mesh stress intensity factors resulted from the finite element computations with the same types of elements and mesh density but for different models. We can even compare them with the result of the finite element modelling of a laboratory experiment and thus obtain the empirical criterion of local failure which will however be expressed in terms of a mesh-dependent quantity. The following section will consider an example of the use of this methodology.

\section{Stress singularity at a toe of a pit}

Consider a 2D elastic model an open pit of a simple configuration whose left part is shown in Figure 2 (the right part is symmetrical). The obvious point of stress singularity is the toe of the excavation, as shown by a small circle in Figure 2. For the model we have chosen the following values of the geometric parameters: $H=200 \mathrm{~m}, B=200,400$ and $600 \mathrm{~m}$. This gives us three geometries which have different angles at the toe and consequently, three different singularity exponents for the stress distribution at the toe. In addition we have chosen the following parameters: $\gamma=23000 \mathrm{~N} / \mathrm{m}^{3}$ and the magnitude of horizontal in situ stress $q=1 \mathrm{~Pa}$ (almost zero). We assumed that the self weight of rock is isotropic; subsequently the elastic moduli do not enter the elastic solution for the boundary conditions chosen).

Three different meshes have been considered. To ensure mesh scalability both minimum and maximum element size have been controlled as well as the shape of the element at the corner (with this control we ensure that the method evaluates the scalable quantities on self-similar regions independently of the element size). Thus, $h=0.001,0.002$ and $0.003 \mathrm{~m}$ have been considered as a minimum size at the corner while the corresponding maximum size on the surrounding region are 10, 20 and $30 \mathrm{~m}$ respectively, see Figure 3 and Tables 1 to 3 . 


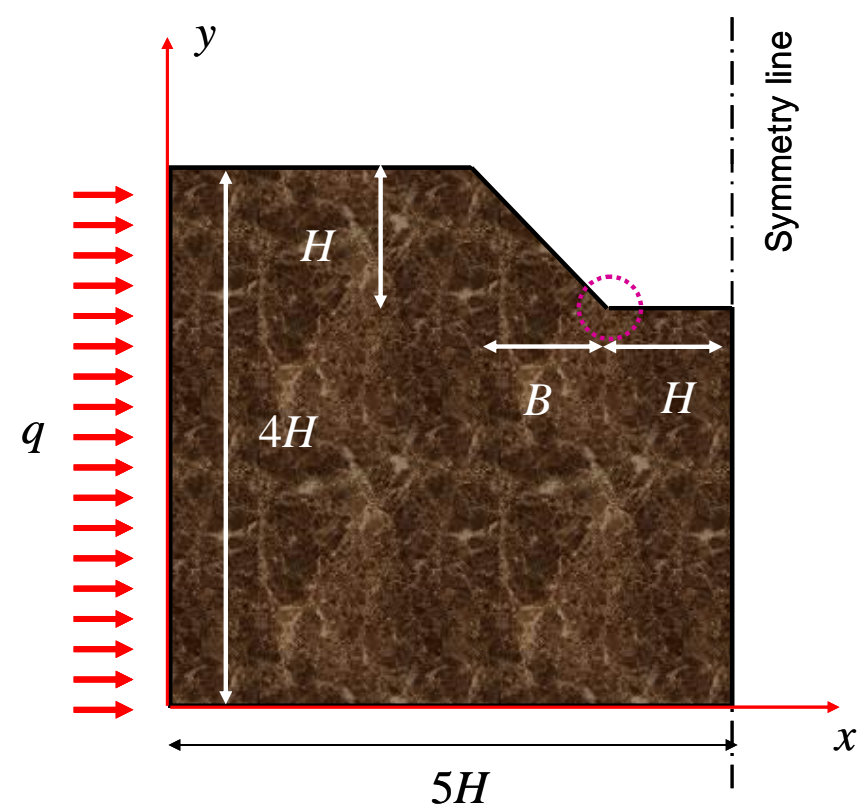

Figure 2 An example of 2D model of an open pit

Table 1 Mesh properties for $B=200 \mathrm{~m}$

\begin{tabular}{llll}
\hline$B=200 \mathrm{~m}$ & $h=0.001-10 \mathrm{~m}$ & $h=0.002-20 \mathrm{~m}$ & $h=0.003-30 \mathrm{~m}$ \\
\hline Nodes & 142962 & 67983 & 45591 \\
Elements & 265297 & 125651 & 84305 \\
\hline
\end{tabular}

Table 2 Mesh properties for $B=400 \mathrm{~m}$

\begin{tabular}{llll}
\hline$B=400 \mathrm{~m}$ & $h=0.001-10 \mathrm{~m}$ & $h=0.002-20 \mathrm{~m}$ & $h=0.003-30 \mathrm{~m}$ \\
\hline Nodes & 141970 & 68296 & 46196 \\
Elements & 263307 & 126275 & 85514 \\
\hline
\end{tabular}

Table 3 Mesh properties for $B=600 \mathrm{~m}$

\begin{tabular}{llll}
\hline$B=600 \mathrm{~m}$ & $h=0.001-10 \mathrm{~m}$ & $h=0.002-20 \mathrm{~m}$ & $h=0.003-30 \mathrm{~m}$ \\
\hline Nodes & 140870 & 68553 & 46242 \\
Elements & 261091 & 126781 & 85600 \\
\hline
\end{tabular}




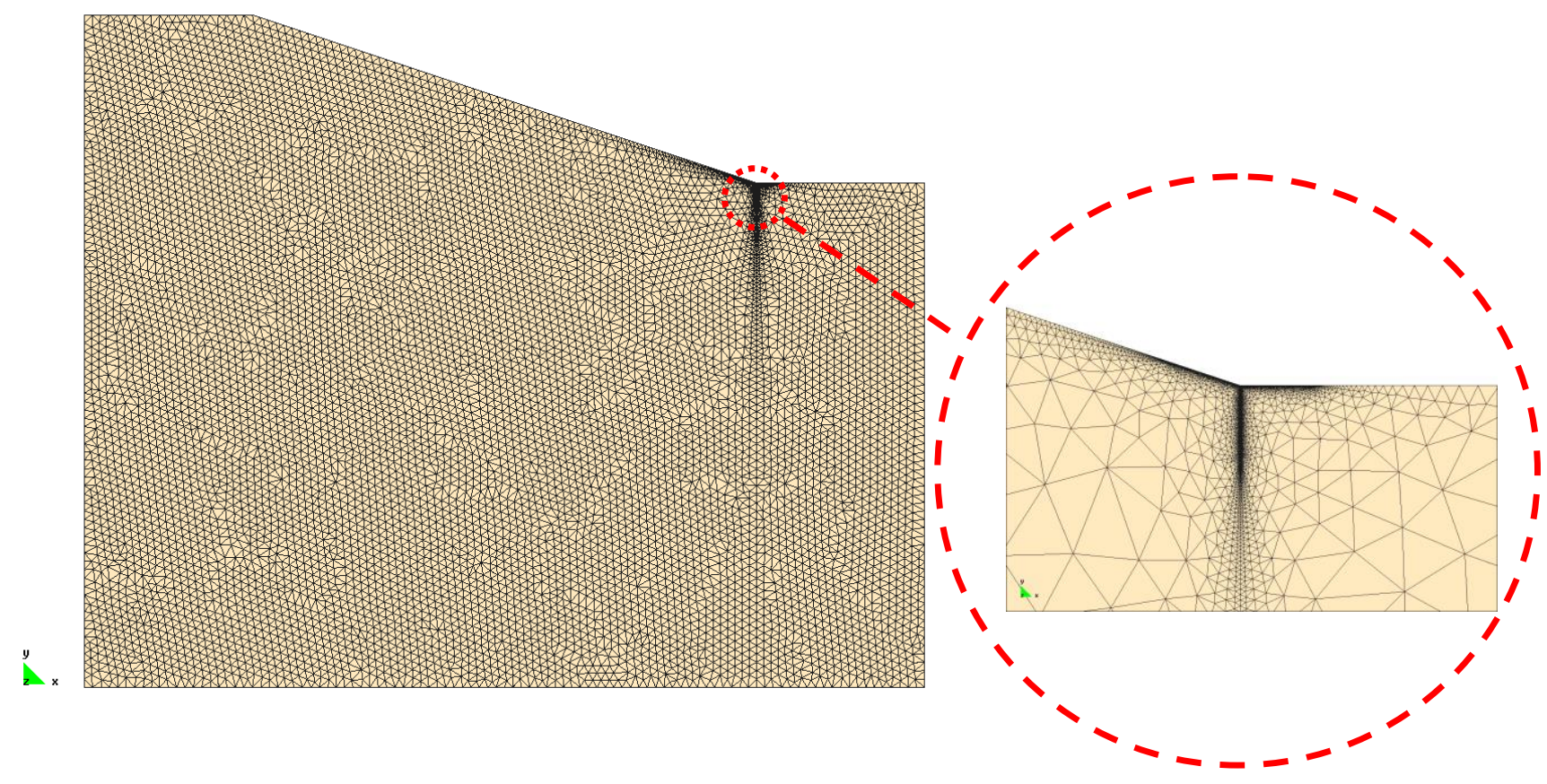

Figure 3 Finite element mesh for the example from Figure 2

Table 4 shows the values of the exponent and pre-factor obtained in the calculations of the maximum principal stress together with the theoretical values of the exponent determined from the angle of the wedge formed by the slope and the bottom of the pit using the results by Severyn and Molski (1996). One can see that the accuracy of recovery of the exponent is quite good. As the analytical solutions for the singularity exponents are not universally available, the mesh scalability concept can deliver a direct method of their determination.

Table 4 Results of modelling for example from Figure 2

\begin{tabular}{lllll}
\hline$B, \mathrm{~m}$ & $\alpha_{\text {Found }}$ & $\alpha_{\text {Theor }}$ & $\delta \%$ & $N_{c}, \mathrm{~Pa} \cdot \mathrm{m}^{-\alpha}$ \\
\hline 600 & -0.320 & -0.322 & -0.50 & $8.97 \mathrm{E}+07$ \\
400 & -0.218 & -0.220 & -0.77 & $9.42 \mathrm{E}+07$ \\
200 & -0.170 & -0.176 & -3.41 & $8.11 \mathrm{E}+07$ \\
\hline
\end{tabular}

Figure 4 shows the comparison between the calculated values of the maximum principal stress at the point of stress singularity (points) and the power law with the parameters from Table 4 (solid lines). It is seen that the power law has already been reached and hence the mesh is scalable. 


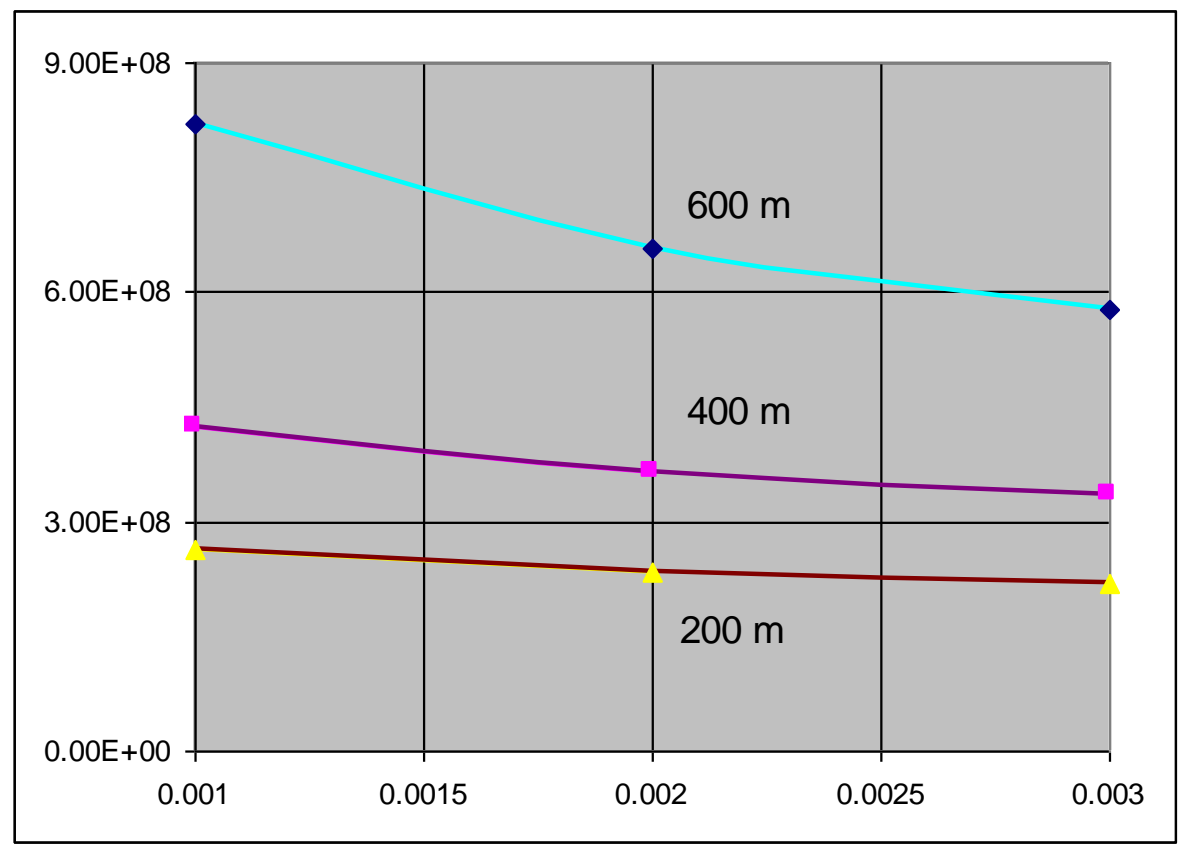

\section{Figure 4 Maximum principal stress at the singularity points (data points) and the power law with parameters listed in Table 4}

We now turn our attention to the mesh stress intensity factors $N_{c}$. In order to illustrate the capacity in utilisation of the information they contain we consider an example of non-uniform rock. We consider the configuration from Figure 2 with $B=200 \mathrm{~m}$. Two different layers of identical area are assumed to have different specific weight. The upper layer is of height $H_{1}=123.607 \mathrm{~m}$ while the lower layer is $H_{2}=76.393 \mathrm{~m}$ thick. To compare these new cases with the uniform rock's results presented in the previous example, the specific weights of the two layers are chosen in the way that their averaged specific weight is equal to the one used for the uniform rock example. With this geometry, Figure 5, we analyse two different scenarios: the first scenario in which the upper layer is denser; the second scenario in which the lower layer is denser. The new $2 \mathrm{D}$ specific weights are $11500 \mathrm{~N} / \mathrm{m}^{3}$ and $34500 \mathrm{~N} / \mathrm{m}^{3}$, for the light and dense layer respectively. The parameters of the new meshes are given in Table 5.

Table 5 Mesh properties for $B=200 \mathrm{~m}$ and different rock stratums

\begin{tabular}{llll}
\hline$B=200 \mathrm{~m}$ & $h=0.001-10 \mathrm{~m}$ & $h=0.002-20 \mathrm{~m}$ & $h=0.003-30 \mathrm{~m}$ \\
\hline Nodes & 142530 & 68752 & 45169 \\
Elements & 264570 & 127257 & 83507 \\
\hline
\end{tabular}




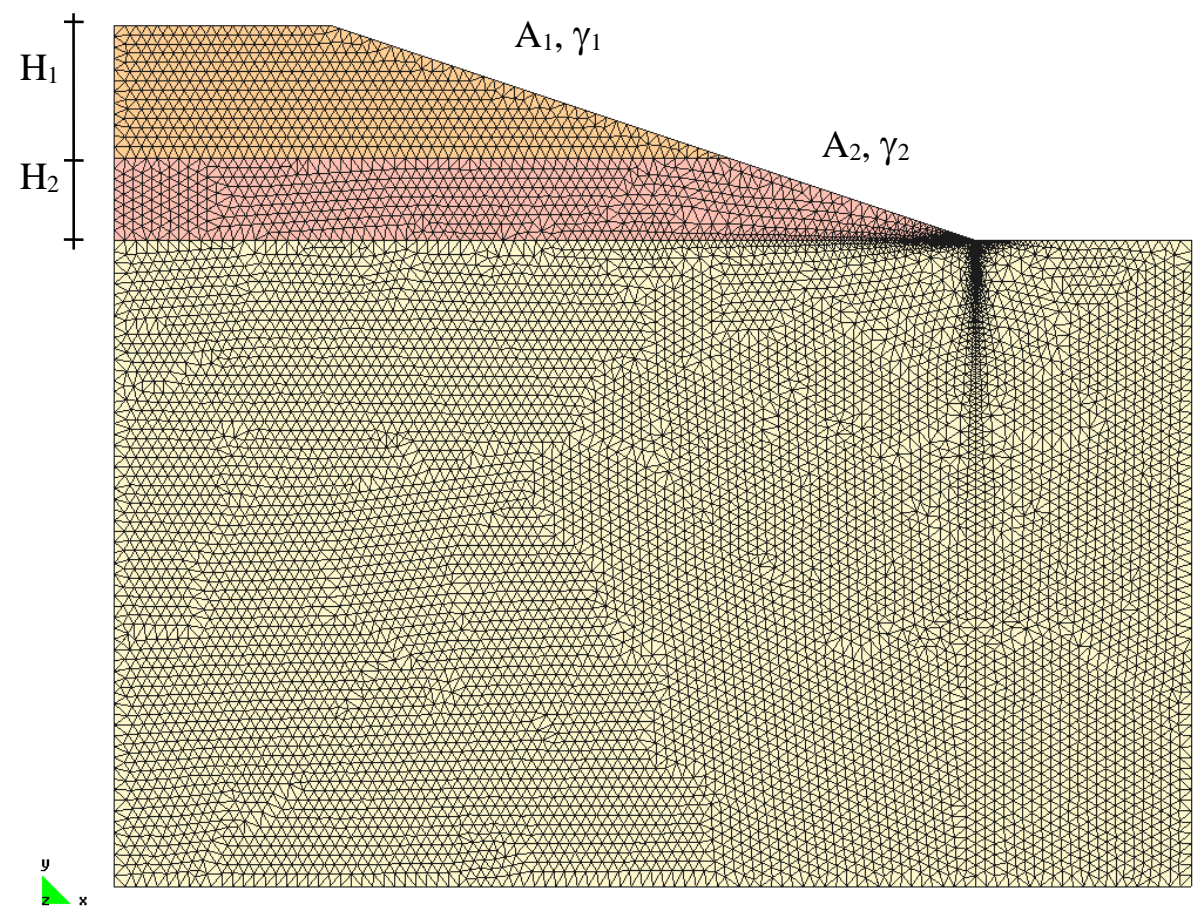

Figure 5 Geometry and mesh for the multilayer example

Table 6 Exponents and pre-factors (mesh stress intensity factors) of scaling of stress concentration for $B=200 \mathrm{~m}$ and different rock stratums

\begin{tabular}{lcc}
\hline Cases & $\alpha$ & $N_{c}, \mathrm{~Pa}^{-\alpha}{ }^{-\alpha}$ \\
\hline Uniform density & -0.164 & $8.31 \mathrm{E}+07$ \\
Lower layer is denser & -0.164 & $8.07 \mathrm{E}+07$ \\
Upper layer is denser & -0.164 & $8.52 \mathrm{E}+07$ \\
\hline
\end{tabular}

It should also be noted that the exponent obtained here for the uniform density is slightly lower than the exponent from Table 4. The reason is that the meshes were different for these cases as evident from Figures 3 and 5. This indicates that strictly speaking we have not yet reached the mesh scalability. Nevertheless given the small (3.5\%) difference between the exponents one can regard the mesh as already being approximately scalable.

We can now compare the mesh stress intensity factors for the three rock mass structures. It is seen that the mesh stress intensity factor, $N_{c}$ is higher for the case when the upper layer is denser. This suggests that the open pit excavated in this type of rock mass would be the least stable. This is followed by the case of homogeneous rock mass and then by the case of denser lower layer. Thus the technique of the scalable mesh allows comparative estimation of the excavation stability without the computing of the actual stresses acting on the representative elements of rock mass. 


\section{Conclusions}

For mesh dependent finite element computations associated with stress singularities we introduced the concept of mesh scalability. The mesh is scalable when the mesh dependent quantities, the stresses at the finite element at the singular point in this case depend upon the element size according to a power law. So when this situation is reached the mesh is fine enough to ensure numerical accuracy; no further refinement is required. The exponent of this dependence gives the value of the singularity exponent in the corresponding elastic solution. The pre-factors, called here the mesh stress intensity factors, can be further used for case studies within the geometries and boundary conditions with the same exponent and the same mesh type. In particular, the lowest values of the mesh stress intensity factors correspond to higher stability of the analysed geotechnical structure.

\section{Acknowledgements}

We acknowledge the financial support from the Australian Computational Earth Systems Simulator (ACcESS) - a Major National Research Facility and the financial support from the Australian Research Council through the Discovery Grant DP0559737.

\section{References}

Barenblatt, G.I. and Botvina, L.R. (1980) Application of the similarity method to damage calculation and fatigue crack growth studies. Defects and Fracture, G.C. Sih and H. Zorski (editors), Martinus Nijhoff Publishers, pp. 71-79.

Bažant, Z.P., Belytschko, T.B. and Chang, T.P. (1984) Continuum theory for strain softening J. Engg. Mech. ASCE, 110, p. 1666.

de Borst, R., Sluys, L.J., Mühlhaus, H.-B. and Pamin, J. (1993) Fundamental issues of finite element analyses of localisation of deformation, Engineering Computations 10, pp. 99-121.

Gelikman, M.B. and Pisarenko, V.F. (1989) About the self-similarity in geophysical phenomena. In: Discrete Properties of the Geophysical Medium, Nauka, Moscow, pp. 109-130.

Klein, P.A., Foulk, J.W., Chen, E.P. Wimmer, S.A. and Gao, H.J. (2001) Physics-based modelling of brittle fracture: cohesive formulations and the application of meshfree methods. Theoretical and Applied Fracture Mechanics, Vol. 37, pp. 99-166.

Pasternak, E., Dyskin, A.V. and Mühlhaus, H.-B. (2001) Stress and failure localization associated with sliding in layered materials, Bifurcation and Localization in Geomechanics. H.-B. Mühlhaus, A.V. Dyskin \& E. Pasternak (editors), Swets \& Zeitlinger, Lisse, pp. 271-278.

Regenauer-Lieb, K. and Yuen, D.A. (2000) Quasi-adiabatic instabilities associated with necking processes of an elastoviscoplastic lithosphere. Physics of Earth and Planetary Interiors, Vol. 118, pp. 89-102.

Severyn, A. and Molski, K. (1996) Elastic stress singularities and corresponding generalized stress intensity factors for angular corners under various boundary conditions. Engineering Fracture Mechanics, 55, No. 4, pp. 529-556.

Sluys, L.J., de Borst, R. and Mühlhaus, H.-B. (1993) Wave propagation, localisation and dispersion in a gradient dependent medium, Int. J. of Solids and Structures, 30, pp. 1153-1171.

Tang C.-A. (1997) Numerical simulation of progressive rock failure and associated seismicity. Int. J. Rock Mech. Min. Sci. 34, No 2, pp. 249-261.

Zosimov, V.V. and Lyamishev, L.M. (1995) Fractals in wave processes. Physics-Uspekhi, 38 (4), pp. $347-384$. 